TRANSACTIONS OF THE

AMERICAN MATHEMATICAL SOCIETY

Volume 331, Number 1, May 1992

\title{
TWO-DIMENSIONAL CREMONA GROUPS ACTING ON SIMPLICIAL COMPLEXES
}

\author{
DAVID WRIGHT
}

\begin{abstract}
We show that the 2-dimensional Cremona group

$$
\mathrm{Cr}_{2}=\operatorname{Aut}_{k} k(X, Y)
$$

acts on a 2-dimensional simplicial complex $C$, which has as vertices certain models in the function field $k(X, Y)$. The fundamental domain consists of one face $F$. This yields a structural description of $\mathrm{Cr}_{2}$ as an amalgamation of three subgroups along pairwise intersections. The subgroup $\mathrm{GA}_{2}=\mathrm{Aut}_{k} k[X, Y]$ (integral Cremona group) acts on $C$ by restriction. The face $F$ has an edge $E$ such that the $G_{2}$ translates of $E$ form a tree $T$. The action of $\mathrm{GA}_{2}$ on $T$ yields the well-known structure theory for $\mathrm{GA}_{2}$ as an amalgamated free product, using Serre's theory of groups acting on trees.
\end{abstract}

\section{INTRODUCTION}

1.1. This discussion sheds light on the relationship between two well-understood automorphism groups. One is the group of $k$-automorphisms of the polynomial ring $k[X, Y]$, for $k$ is a field. This is often viewed anti-isomorphically as the group of algebraic automorphisms of the affine plane $\mathbb{A}_{k}^{2}$. The other group consists of the $k$-automorphisms of the rational function field $k(X, Y)$; elements of this group corresponds to birational automorphisms of $\mathbb{A}_{k}^{2}$, or equivalently, of the projective plane $\mathbb{P}_{k}^{2}$.

1.2. Sections 2 and 3 summarize existing knowledge of these groups and present them as free products with amalgamation (Theorems 2.4 and 3.11). For Aut $_{k} k[X, Y]$ this draws from the classical theorem of Jung and Van der Kulk, which asserts that this group is generated by the set of elements which are of linear or elementary type, and the theorem of Nagata which describes the group as an amalgamated free product of two groups. For $\mathrm{Aut}_{k} k(X, Y)$, the classical Noether's Theorem asserts that, for $k$ algebraically closed, this group is generated by the linear fractional transformations together with the standard quadratic transformation. However, our conclusions are based on recent results of Iskovskikh which give a set of defining relations of $\operatorname{Aut}_{k} k(X, Y)$ in terms of generators slightly different from those of Noether's Theorem. From Iskovskikh's generators and relations, we deduce that this group is the free product of three subgroups amalgamated along pairwise intersections.

Received by the editors August 25, 1989 and, in revised form, February 16, 1990.

1980 Mathematics Subject Classification (1985 Revision). Primary 14J50, 13B10, 13B35, 05C05, $20 \mathrm{~F} 05$. 
1.3. Although Aut $_{k} k(X, Y)$ contains Aut $_{k} k[X, Y]$ as a subgroup, the relationship between the structures of these two groups has long been a mystery. For although the proofs which yield generators and relations for the two groups seem related in that they both involve the technique of blowing up points of indeterminacy, it seems that neither theorem can be deduce from the other. The purpose of this paper, then, is to present a framework which unifies the two structures. The method is suggested by the fact that both groups admit a description as amalgamated free products. Topologists have long recognized that such a structure is tantamount to an action of the group on a simply connected simplicial complex. We will exhibit these complexes in such a way that their vertices correspond to certain "multiprojective spaces" having function field $k(X, Y)$. The complex on which $\operatorname{Aut}_{k} k(X, Y)$ acts contains the one for $\operatorname{Aut}_{k} k[X, Y]$ in a way compatible with the containment $\operatorname{Aut}_{k} k(X, Y) \supset \operatorname{Aut}_{k} k[X, Y]$; moreover there is containment between suitably chosen fundamental domains for the respective actions.

We begin by introducing some notation and stating the theorems. As above, $k$ will be a field; we denote by $k^{*}$ the set of nonzero elements of $k$.

\section{The AUTOMORPHISM GROUP OF $k[X, Y]$}

2.1. Integral Cremona group. Let $\mathrm{GA}_{2}(k)$, or just $\mathrm{GA}_{2}$, denote the group of $k$-automorphisms of the polynomial ring $k[X, Y]$. This is called the integral Cremona group.

Letting $W=\operatorname{Spec} k[X, Y]$, we see that elements of $\mathrm{GA}_{2}$ correspond antiisomorphically to automorphisms of the variety $W$. In $\S 4$ our dicussion will involve different models in $k(X, Y)$ which contain a fixed $\mathbb{A}_{k}^{2}$ as a Zariski open set, and we will let this fixed $\mathbb{A}_{k}^{2}$ be $W$. We will refer to $W$ as the standard $\left.\mathbb{A}^{2}\right)$ (in $\left.k(X, Y)\right)$.

2.2. Vector representation. An element $\varphi$ of $\mathrm{GA}_{2}$ can be represented as a pair of polynomials $(F, G)$, where $F=\varphi(X), G=\varphi(Y)$.

2.3. Linear and triangular elements. We denote by $A f$ the subgroup of $\mathrm{GA}_{2}$ consisting of those elements $\varphi=(F, G)$ for which $F$ and $G$ have total degree one in $X$ and $Y$ (but are not necessarily homogeneous). form

We let $B A$ be the subgroup of $\mathrm{GA}_{2}$ consisting of all $\varphi=(F, G)$ of the

$$
F=a X+b, \quad G=c Y+g(X),
$$

where $a, c \in k^{*}, b \in k$, and $g(X) \in k[X]$. Elements of this subgroup are called triangular, since these are precisely the automorphisms which preserve the containment $k[X] \subset k[X, Y]$. It is clear that the intersection of $A f$ and $B A$, which we denote by $B$, consists of those $\varphi=(F, G)$ which are of the form (1) where $g(X)$ has degree $\leq 1$.

The well-known structure theorem for $\mathrm{GA}_{2}$ is

2.4. Theorem. $\mathrm{GA}_{2}$ has the amalgamated free product structure

$$
\mathrm{GA}_{2}=A f *_{B} B A \text {. }
$$

(A proof will be given in $\S 4$.) 
2.5. Remarks on the origin of this theorem. That $\mathrm{GA}_{2}$ is generated by $A f$ and $B A$ was first proved by Jung [10] for $k$ of characteristic zero. Van der Kulk [19] generalized this to arbitrary characteristic and proved a factorization theorem which essentially gives the amalgamated free product structure, although he did not state it in this language. Nagata [12] seems to be the first to have stated and proved the assertion as it appears above. The techniques in these proofs require that $k$ be algebraically closed. However, it is not hard to deduce the general case from this (see [20]).

Some fairly recent proofs have been given which use purely algebraic techniques, and for which it is not necessary to assume $k$ is algebraically closed [3], [11].

\section{THE AUTOMORPHISM GROUP OF $k(X, Y)$}

3.1. Cremona group. Let $\mathrm{Cr}_{2}$ denote the group of $k$-automorphisms of the field $k(X, Y)$. It will be called the (full) Cremona group. This group is antiisomorphic to the group of birational automorphisms of the projective (or affine) plane. (It should be noted that most sources use the term "Cremona group" and the symbol $\mathrm{Cr}$ to refer to the group of birational automorphisms.) Note that $\mathrm{Cr}_{2}$ contains the integral Cremona group $\mathrm{GA}_{2}$ as a subgroup.

3.2. Homogeneous and nonhomogeneous vector representations. An element $\varphi$ $\in \mathrm{Cr}_{2}$ can be represented by the pair of rational functions $(F, G)$, where $F=\varphi(X), G=\varphi(Y)$.

Another way to realize elements of $\mathrm{Cr}_{2}$ is as follows: Letting $X=x / z$ and $Y=y / z, k(X, Y)$ becomes the field of homogeneous rational functions of degree zero in $k(x, y, z)$. Given $\varphi=(F, G) \in \mathrm{Cr}_{2}$, write $F=F_{0} / H_{0}$, $G=G_{0} / H_{0}$, where $F_{0}, G_{0}$, and $H_{0}$ are polynomials in $X$ and $Y$. Now replace $X, Y$ by $x / z, y / z$ and homogenize to get $F=f(x, y, z) / h(x, y, z)$, $G=g(x, y, z) / h(x, y, z)$, where $f, g$ and $h$ are forms of the same degree. Then $\varphi$ is represented by the triple $(f: g: h)$, uniquely up to common factors of $f, g$, and $h$. We may take $f, g$, and $h$ to have no common factors. They serve as the coordinate functions for the birational automorphism of $\mathbb{P}^{2}$ determined by $\varphi$.

We will be shifting back and forth between these representation of $\varphi$. The representation $\varphi=(f: g: h)$ will be called the homogeneous representation; the representation $\varphi=(F, G)$ will be called nonhomogeneous.

3.3. Linear fractional transformations. The group $\mathrm{PGL}_{3}(k)$ is contained as a subgroup of $\mathrm{Cr}_{2}$, whereby the class of the matrix

$$
\left(\begin{array}{lll}
a & a^{\prime} & a^{\prime \prime} \\
b & b^{\prime} & b^{\prime \prime} \\
c & c^{\prime} & c^{\prime \prime}
\end{array}\right),
$$

is identified with the Cremona transformation having homogeneous representation

$$
\left(a x+b y+c z: a^{\prime} x+b^{\prime} y+c^{\prime} z: a^{\prime \prime} x+b^{\prime \prime} y+c^{\prime \prime} z\right),
$$

and nonhomogeneous representation

$$
\left(\frac{a X+b Y+c}{a^{\prime \prime} X+b^{\prime \prime} Y+c^{\prime \prime}}, \frac{a^{\prime} X+b^{\prime} Y+c^{\prime}}{a^{\prime \prime} X+b^{\prime \prime} Y+c^{\prime \prime}}\right) .
$$


(Note: it may seem to the reader that we should have taken the transpose of the above matrix. But recall that we are viewing Cremona transformations as field automorphisms, not as transformations of $\mathbb{P}^{2}$.) Such elements are sometimes called linear fractional transformations.

Since $k(X, Y)$ can be identified as the function field of $\mathbb{P}_{k}^{2}$ in many ways, let Proj $k[x, y, z]$, where $X=x / z, Y=y / z$, be called the standard $\mathbb{P}^{2}$ (in $k(X, Y))$. This surface contains the standard $\mathbb{A}^{2}, W$ (see 2.1), the complement of $W$ being the line $z=0$, which we will call the line at infinity and denote $l_{\infty}$. Note that the linear fractional transformations correspond to the actual automorphisms (not just birational automorphisms) of the standard $\mathbb{P}^{2}$.

3.4. Standard quadratic transformation. Another familiar element of $\mathrm{Cr}_{2}$ is the standard quadratic transformation $\sigma$, given by $(y z: x z: x y)=(1 / X, 1 / Y)$. Clearly $\sigma$ has order 2 . The birational automorphism of $\mathbb{P}_{k}^{2}$ corresponding to $\sigma$ can be explained as the blow-up of the points $(1: 0: 0),(0: 1: 0),(0: 0: 1)$ followed by the blow-down of the proper transforms of the $x, y$, and $z$ axes.

3.5. Noether's Theorem. For $k$ algebraically closed, $\mathrm{Cr}_{2}$ is generated by $\mathrm{PGL}_{3}(k)$ together with the standard quadratic transformation $\sigma$.

3.6. Remarks. This result bears the name "Noether's Theorem" because it was first claimed by Max Noether [14], although his proof was flawed. The first correct proof seems to have been given by Castelnuovo [2]; another early proof appears in [6]. More recent proofs, with modern terminology, can be found in [16] and [13]. These proofs all assume $k$ is algebraically closed, and unlike the Jung-Van der Kulk-Nagata Theorem (Theorem 2.3), this hypothesis is essential, according to some new results of Iskovskikh.

3.7. The standard $\mathbb{P}_{k}^{1} \times \mathbb{P}_{k}^{1}$ and its automorphism group. There is a unique model in $k(X, Y)$ isomorphic to $\mathbb{P}_{k}^{1} \times \mathbb{P}_{k}^{1}$ whose projection maps correspond to the inclusions $k(X) \rightarrow k(X, Y)$ and $k(Y) \rightarrow k(X, Y)$. We will call this model the standard $\mathbb{P}^{1} \times \mathbb{P}^{1}$ (in $\left.k(X, Y)\right)$.

The subgroup of $\mathrm{Cr}_{2}$ which corresponds to the automorphisms of the standard $\mathbb{P}^{1} \times \mathbb{P}^{1}$ is $\left(\mathrm{PGL}_{2}(k) \times \mathrm{PGL}_{2}(k)\right) \rtimes\langle\tau\rangle$, where

$$
\left(\left(\begin{array}{ll}
a & c \\
b & d
\end{array}\right),\left(\begin{array}{ll}
a^{\prime} & c^{\prime} \\
b^{\prime} & d^{\prime}
\end{array}\right)\right) \in \mathrm{PGL}_{2}(k) \times \mathrm{PGL}_{2}(k)
$$

is identified with the element

$$
\left(\frac{a X+b}{c X+d}, \frac{a^{\prime} Y+b^{\prime}}{c^{\prime} Y+d^{\prime}}\right) \in \mathrm{Cr}_{2},
$$

and $\tau=(Y, X)$. This group will play a key role in our structure theorem for $\mathrm{Cr}_{2}$.

3.8. Triangular subgroups. We view $k(X, Y)$ as $k(X)(Y)$ and consider elements of $\mathrm{Cr}_{2}$ which fix $k(X)$. This subgroup is identified with $\mathrm{PGL}_{2}(k(X))$, where the matrix class

is identified with

$$
\left(\begin{array}{ll}
a(X) & c(X) \\
b(X) & d(X)
\end{array}\right) \in \operatorname{PGL}_{2}(k(X)),
$$

$$
\left(X, \frac{a(X) Y+b(X)}{c(X) Y+d(X)}\right) \in \mathrm{Cr}_{2} .
$$


Note that this subgroup contains the element $\varepsilon=(X, X / Y)=(x y: x z: y z)$. This automorphism appears in Iskovskikh's Theorem, stated below.

Let us note that a $k$-automorphism of $k(X)$, which can be identified with an element of $\mathrm{PGL}_{2}(k)$, gives rise to a unique automorphism of $k(X, Y)$ which fixes $Y$. In this way, $\mathrm{PGL}_{2}(k)$ is identified with the subgroup of elements of $\mathrm{Cr}_{2}$ of the form

$$
\left(\frac{a X+b}{c X+d}, Y\right)
$$

where $\left(\begin{array}{ll}a & b \\ c & d\end{array}\right) \in \mathrm{PGL}_{2}(k)$.

Letting $\mathrm{PGL}_{2}(k)$ act on $k(X)$ gives rise to a semidirect product

$$
\mathrm{PGL}_{2}(k) \ltimes \mathrm{PGL}_{2}(k(X)) \text {. }
$$

An element

$$
\left(\left(\begin{array}{ll}
a & c \\
b & d
\end{array}\right),\left(\begin{array}{ll}
a^{\prime}(X) & c^{\prime}(X) \\
b^{\prime}(X) & d^{\prime}(X)
\end{array}\right)\right)
$$

in this group is identified with the element

$$
\left(\frac{a X+b}{c X+d}, \frac{a^{\prime}(X) Y+b^{\prime}(X)}{c^{\prime}(X) Y+d^{\prime}(X)}\right) \in \mathrm{Cr}_{2},
$$

and this is the subgroup of $\mathrm{Cr}_{2}$ which preserves the containment $k(X) \subset$ $k(X, Y)$. Its elements are called triangular automorphisms. Note that this group contains the direct product $\mathrm{PGL}_{2}(k) \times \mathrm{PGL}_{2}(k)$, which contains the standard quadratic automorphism $\sigma=(1 / X, 1 / Y)$.

Note also that this subgroup corresponds to the automorphism group of $\mathbb{P}_{k(X)}^{1}$. We will refer to the $k$-scheme $\mathbb{P}_{k(X)}^{1}$ as the standard $\mathbb{P}^{1}$ (in $k(X, Y)$ ).

3.9. The group $G^{(n)}$. Crucial to our dicussion in $\S \S 4$ and 5 are the groups $G^{(n)}, n \geq 1$, defined by

$$
G^{(n)}=\left\{\left(\frac{a X+b}{c X+d}, \frac{t Y+f(X)}{(c X+d)^{n}}\right) \mid\left(\begin{array}{ll}
a & b \\
c & d
\end{array}\right) \in \mathrm{GL}_{2}(k), t \in k^{*}, \operatorname{deg} f \leq n\right\}
$$

Note that $G^{(n)}$ is contained in the triangular subgroup of $\mathrm{Cr}_{2}$. Elements of $G^{(n)}$ correspond to automorphisms of the classical surface $F_{n}$, as described below.

3.10. The surfaces $F_{n}$. The reader is referred to [13] or [16] for a full discussion of these surfaces. Briefly, $F_{n}$ is a smooth rational surface for $n \geq 0$, minimal if $n \neq 1$, with $F_{0} \cong \mathbb{P}_{k}^{1} \times \mathbb{P}_{k}^{1}$. There is a map $\pi: F_{n} \rightarrow \mathbb{P}_{k}^{1}$ by which $F_{n}$ is a $\mathbb{P}_{k}^{1}$-bundle over $\mathbb{P}_{k}^{1}$. For $n \geq 1, \pi$ is unique and $F_{n}$ contains a special section $\Delta_{n}$ having the property that $\left(\Delta_{n}^{2}\right)=-n$. This curve $\Delta_{n}$ is the only prime divisor in $F_{n}$ having negative self-intersection. All fibers of $\pi$ are linearly equivalent, and if $f$ is such a fiber we have $\left(f^{2}\right)=0,\left(f, \Delta_{n}\right)=1, \operatorname{Pic} F_{n}=\mathbb{Z} \cdot[f] \oplus \mathbb{Z} \cdot\left[\Delta_{n}\right]$ (the free abelian group on the divisor classes of $f$ and $\Delta_{n}$ ). Given a point $p \in F_{n}$, there is an elementary transformation $\operatorname{elm}_{p}$ centered at $p$ which transforms $F_{n}$ to an $F_{n+1}$ or an $F_{n-1}$. This consists of first blowing up at $p$, then blowing down the proper transform of the fiber containing $p$. If $p \in \Delta_{n}$, the resulting surface is an $F_{n+1}$; if not (and $n \geq 1$ ) it is an $F_{n-1}$. 
3.11. The standard $F_{n} . F_{n}$ can are realized as follows. Let

$$
\begin{gathered}
W=\operatorname{Spec} k[X, Y] \quad\left(\text { the standard } \mathbb{A}^{2}(\text { see } 2.1)\right), \\
V=\operatorname{Spec} k\left[X, Y^{-1}\right], \quad W^{\prime}=\operatorname{Spec} k\left[X^{-1}, Y^{\prime}\right], \quad V^{\prime}=\operatorname{Spec} k\left[X^{-1}, Y^{\prime-1}\right],
\end{gathered}
$$

where $Y^{\prime}=X^{-n} Y$. then $W \cup V \cup W^{\prime} \cup V^{\prime} \cong F_{n}$. The complement of $W \cup W^{\prime}$ is $\Delta_{n}$, and the map $\pi$ arises from the containment $k(X) \rightarrow k(X, Y)$. We call this model the standard $F_{n}$ (in $k(X, Y)$ ). Note that for $n=0$ this is the standard $\mathbb{P}^{1} \times \mathbb{P}^{1}$ (see 3.8). The complement of $W$ in the standard $F_{n}$ is the union of $\Delta_{n}$ and one fiber of $\pi$, the latter of which we call the fiber at infinity (with respect to $W$ ) and denote by $f_{n, \infty}$. Thus we have

$$
F_{n}=W \cup \Delta_{n} \cup f_{n, \infty} .
$$

For $n=1$, this surface is obtained from the standard $\mathbb{P}^{2}$ (see 3.3) by blowing up the point $(0: 1: 0)$. The resulting exceptional curve becomes $\Delta_{1}$ and the proper transform of $l_{\infty}$ (see 3.3) becomes $f_{1, \infty}$. If we perform the elementary transformation $\operatorname{elm}_{p}$ on the standard $F_{n}$, where $p=\Delta_{n} \cap f_{n, \infty}$, we obtain the standard $F_{n+1}$.

For $n \geq 1$, any automorphism of $F_{n}$ must preserve $\Delta_{n}$, since this curve is characterized by the property $\Delta_{n}^{2}=-n$. An easy argument using intersection numbers shows that any such automorphism must carry fibers (of $\pi$ ) to fibers. From these facts one can show without too much difficulty that, for the standard $F_{n}$, such automorphisms correspond precisely to elements of $G^{(n)}$, so that $G^{(n)}$ is anti-isomorhic to $\operatorname{Aut}\left(F_{n}\right)$.

An important breakthough was recently made by V. A. Iskovskikh, who proved the following:

3.12. Theorem (Iskovskikh). $\mathrm{Cr}_{2}$ is generated by the triangular group $\mathrm{PGL}_{2}(k)$ $\ltimes \mathrm{PGL}_{2}(k(X))$ together with the element $\tau=(Y, X)$. Moreover, a complete set of relations is given by the group law of the triangular group, $\tau^{2}=1$, and the relations

(*) $\tau \cdot(F, G) \cdot \tau=(G, F)$, for all $(F, G) \in \mathrm{PGL}_{2}(k) \times \mathrm{PGL}_{2}(k)$;

$(* *)(\tau \varepsilon)^{3}=\sigma$ (where, as above, $\varepsilon=\left(X, \frac{X}{Y}\right)$ and $\left.\sigma=\left(\frac{1}{X}, \frac{1}{Y}\right)\right)$.

(See [8] and [9].)

(We will comment in 3.14 on the relationship between this theorem and Noether's Theorem.)

We now state a structure theorem, based on Iskovskikh's result, which presents $\mathrm{Cr}_{2}$ as a product with amalgamations along pairwise intersections.

3.13. Theorem. Let

$$
\begin{aligned}
& A_{1}=\mathrm{PGL}_{3}(k), \\
& A_{2}=\left(\mathrm{PGL}_{2}(k) \times \mathrm{PGL}_{2}(k)\right) \rtimes\langle\tau\rangle, \\
& A_{3}=\mathrm{PGL}_{2}(k) \ltimes \mathrm{PGL}_{2}(k(X)),
\end{aligned}
$$

(each identified as a subgroup of $\mathrm{Cr}_{2}$ as described in 3.3, 3.7, and 3.8). Then $\mathrm{Cr}_{2}$ is the free product of $A_{1}, A_{2}$, and $A_{3}$ amalgamated along their pairwise intersections in $\mathrm{Cr}_{2}$.

Proof. The proof appeals to Iskovskikh's Theorem (3.12). Let $G$ be the group obtained by amalgamating $A_{1}, A_{2}$, and $A_{3}$ along their pairwise intersections 
in $\mathrm{Cr}_{2}$. We clearly have a group homomorphism $\alpha: G \rightarrow \mathrm{Cr}_{2}$ restricting to the identity on $A_{1} \cup A_{2} \cup A_{3}$. The map $\alpha$ is surjective, since the image contains $A_{3}$ and $\tau\left(\in A_{2}\right)$, which generate $\mathrm{Cr}_{2}$ according to Iskovskikh's Theorem.

We now wish to define a homomorphism $\beta: \mathrm{Cr}_{2} \rightarrow G$. According to Iskovskikh's Theorem, $\mathrm{Cr}_{2}$ is generated by $A_{3}$ and $\{1, \tau\}$, the latter lying inside $A_{2}$, so we have a map $\tilde{\beta}$ from the free product $\{1, \tau\} * A_{3}$ to $G$. We must show that the relations $(*)$ and $(* *)$ of Theorem 3.12 hold in $G$.

Note that $(*)$ obviously holds, since it takes place in $A_{2}$.

As for $(* *)$, note that the equality $\varepsilon=\rho \sigma$, where $\rho=(1 / X, Y / X)$, holds in $A_{3}$, hence in $G$. Since $\sigma$ and $\rho$ commute in $A_{3}$, they commute in $G$. Since $\sigma$ and $\tau$ commute in $A_{2}$, they commute in $G$. Thus we have the following equations in $G$ :

$$
(\tau \varepsilon)^{3}=(\tau \rho \sigma)^{3}=(\tau \rho)^{3} \sigma^{3} .
$$

Observe that $\tau$ and $\rho$ lie in $A_{1}$, and that $(\tau \rho)^{3}=1$ in $A_{1}$, hence in $G$. Since $\sigma^{3}=\sigma$ (in $A_{2}$, hence in $G$ ), it follows from (1) that $(\tau \varepsilon)^{3}=\sigma$ in $G$, as desired.

Hence $\tilde{\beta}$ induces a map $\beta: \mathrm{Cr}_{2} \rightarrow G$ which restricts to the identity on $A_{3}$ and $\{1, \tau\}$. Since $\mathrm{Cr}_{2}$ is generated by $A_{3}$ and $\{1, \tau\}$, it is clear that $\alpha \circ \beta=1$. It will follow that $\alpha$ is an isomorphism (with $\alpha^{-1}=\beta$ ) once we show that $\beta$ is surjective.

To see that $\beta$ is surjective, note that its image contains $A_{3} \subset G$ and $\tau \in$ $A_{1} \cap A_{2} \subset G$. It is an easy exercise to see that both $A_{1}$ and $A_{2}$ are generated by their intersection with $A_{3}$ (in $\mathrm{Cr}_{2}$ ) together with $\tau$. Therefore $A_{1}$ and $A_{2}$ are in the image of $\beta$, and since $G$ is generated by $A_{1} \cup A_{2} \cup A_{3}, \beta$ is surjective. The theorem is proved.

3.14. Remark. One easily proves that $A_{2}$ is generated by its intersection with $A_{1}$ together with $\sigma$. It can be shown (though not so easily) that $A_{3}$ is also generated by its intersection with $A_{1}$ together with $\sigma$. (This is done by Iskovskikh in [7].) Thus, from Iskovskikh's results one can deduce Noether's Theorem (3.5), which says that $\mathrm{Cr}_{2}$ is generated by $A_{1}$ together with $\sigma$.

\section{TREE ACTIONS WHICH YIELD THE STRUCTURE THEOREM FOR GA}

4.1. Tree theory. The fact that $\mathrm{GA}_{2}$ has a decomposition as an amalgamated free product (Theorem 2.4) says that it acts without inversion on a tree (see [15]). Such a tree can be constructed abstractly, but it is more useful to realize it in a natural context. This was done by Roger Alperin [1] (see also [3]), where the vertices of the tree correspond to certain subvector spaces of $k[X, Y]$. This section will culminate in a realization of what is essentially Alperin's tree, but our approach is closer in spirit to that of Gizatullin and Danilov [4] and [5], which is to consider the $\mathrm{GA}_{2}$ action on a tree whose vertices correspond to certain smooth compactifications of $\mathbb{A}^{2}$.

4.2. Let $k$ be an algebraically closed field. Any separated reduced, irreducible $k$-scheme whose function field is $k(X, Y)$ can be identified with collection of local rings in $k(X, Y)$ corresponding to its points. Thus $\mathrm{Cr}_{2}$ (and $\mathrm{GA}_{2}$ ) acts on the set of such $k$-schemes.

4.3. $W$-admissible models. Let $W$ be the standard $\mathbb{A}^{2}$ (see 2.1). As above, we identify $W$ with the set of local rings in $k(X ; Y)$ corresponding to its 
points; these will be localizations of $k[X, Y]$. Let $S$ be a complete, nonsingular surface containing $W$ as a Zariski open subset. We will say that $S$ is a $W$ admissible model if $S$ satisfies one of these (mutually exclusive) conditions:

(1) $S \cong \mathbb{P}_{k}^{2}$ (in which case it is automatic that $S=W \cup l$, where $l$ is the "line at infinity" with respect to $W$ ), or

(2) $S \cong F_{n}$ for some integer $n \geq 1$, and $S=W \cup \Delta_{n} \cup f_{n}$, where $F_{n}$ is the classical surface described in $3.11, \Delta_{n}$ is its special section, and $f_{n}$ is a fiber of the canonical map $\pi: F_{n} \rightarrow \mathbb{P}_{k}^{1}$.

If the first condition holds, we say that $S$ is $a \mathbb{P}^{2}$ (in $k(X, Y)$; if the second condition holds for some $n$, we say $S$ is an $F_{n}$ (in $k(X, Y)$ ). Note that any $W$-admissible model $S$ is a relatively minimal model (in the sense of [16]) unless $S$ is an $F_{1}$.

4.4. The graph $\widetilde{T}$ (of $W$-admissible models). For $W \cong \mathbb{A}^{1}$ as in 4.3 , we construct a graph $\widetilde{T}$ whose vertices consist of all $W$-admissible models. An $F_{n}$ and an $F_{n+1}$ are connected by an edge if the $F_{n+1}$ is obtained from the $F_{n}$ by means of the elementary transformation $\operatorname{elm}_{p}$ (see 3.11) with center $p=f_{n} \cap \Delta_{n}$ $\left(f_{n}\right.$ and $\Delta_{n}$ are as in condition (2) of 4.3). This transformation blows up $p$, then blows down the proper transform of $f_{n}$ (see [16, Chapter $\mathrm{V}, \S 1$ ] or [13, $\S 2]) . \mathrm{A} \mathbb{P}^{2}$ and an $F_{1}$ are connected by an edge if the $F_{1}$ is obtained from the $\mathbb{P}^{2}$ by blowing up a point on $l$ ( $l$ is as in condition (1) of 4.3).

4.5. Adjacency in $\tilde{T}$. Two vertices are called adjacent if they are connected by an edge. Note that there is precisely one $F_{n+1}$ adjacent in $\widetilde{T}$ to a given $F_{n}$. However, for $n \geq 2$, there are many $F_{n+1}$ 's adjacent to a given $F_{n}$, since one such is obtained by performing elm $\operatorname{elm}_{p}$ for any $p$ on $f_{n}$ but not on $\Delta_{n}$. Also note that each $F_{1}$ is adjacent to precisely one $\mathbb{P}^{2}$; but a given $\mathbb{P}^{2}$ is adjacent to many $F_{1}$ 's-one for each point on $l_{\infty}$.

4.6. Type of a vertex. It will be convenient for us to say that a vertex $S$ in $\widetilde{T}$ has type $n$ if $S$ is an $F_{n}(n \geq 1)$, or has type zero if $S$ is a $\mathbb{P}^{2}$. Clearly two adjacent vertices in $\widetilde{T}$ have types which differ by one. According to 4.5, each vertex of type $n \geq 1$ is adjacent to precisely one vertex of type $n+1$.

4.7. Paths. A sequence of vertices $V_{1}, \ldots, V_{r}$ in $\widetilde{T}$ with $V_{i-1}$ adjacent to $V_{i}$ for $i=1, \ldots, r$ determines a path in $\tilde{T}$. We say the path has no backtracks if $V_{i-2} \neq V_{i}$, whenever $2 \leq i \leq r$.

4.8. Lemma. Let $V_{0}, \ldots, V_{r}, r \geq 1$, be a path in $\widetilde{T}$ with no backtracks. Suppose $V_{0}$ is an $F_{1}$ and $V_{1}$ is a $\mathbb{P}^{2}$. Let $O$ be the discrete valuation ring which is the local ring of the special section $\Delta$ in $V_{0}$. Then $O$ dominates the local ring of a closed point of $V_{r}$.

Proof. Note that $V_{1}$ is obtained by blowing down $\Delta$ to a point $q$ on $V_{1}$, and hence $O$ dominates the local ring of $q$. If $r \geq 2, V_{2}$ is an $F_{1}$ obtained by blowing up a point $p$ on $V_{1}$, and since $V_{2} \neq V_{0}$, we have $p \neq q$. Thus $O$ dominates a point on $V_{1}$ which is not the center of the blow-up that yields $V_{2}$.

Let $U_{1}, \ldots, U_{n}$ be the subsequence of $V_{0}, \ldots, V_{r}$ consisting of all its $\mathbb{P}^{2}$ 's. Note that $n \geq 1$, since $U_{1}=V_{1}$. If $n>1$, then, for $i=1, \ldots, n-1$, the path from $U_{i}$ to $U_{i+1}$ has the form

$$
U_{i}, S_{1}, S_{2}, \ldots, S_{m_{i}-1}, S_{m_{i}}=S_{m_{i}}^{\prime}, S_{m_{i}-1}^{\prime}, \ldots, S_{2}^{\prime}, S_{1}^{\prime}, U_{i+1}
$$


with $m_{i} \geq 2$ (since there are no backtracks) and $S_{j}, S_{j}^{\prime} \cong F_{j}$, for $j=$ $1, \ldots, m_{i}$. If $i=n$, the path from $U_{n}$ to $V_{r}$ may be extended past $V_{r}$ to such a path. Hence it suffices to show $O$ dominates the local ring of a closed point on each surface in (1).

We may assume by induction that $O$ dominates a closed point $q$ on $U_{i}$ which is not the center of the blow-up which yields $S_{1}$. (We have observed that this is the case when $i=1$.) Thus $q$ is a point on $S_{1}$ lying on the fiber at infinity (with respect to $W$ ), but not on its special section. One easily sees that the local ring of $q$, and hence $O$, dominates a point of $S_{2}\left(\cong F_{2}\right)$ satisfying the same conditions, and so on up to $S_{m_{i}}$. Moreover, the elementary transformation leading from $S_{m_{i}}$ to $S_{m_{i}-1}^{\prime}$ does not blow up the center of $O$, otherwise we would have a backtrack in the path. Therefore the center of $O$ on $S_{m_{i}-1}^{\prime}$ is the intersection of its special section and its fiber at infinity, and this holds for $S_{m_{i}-2}^{\prime}$ down to $S_{1}^{\prime}$. Thus on $U_{i+1}, O$ dominates a point at infinity which is not the center of the blow-up which yields the next surface along the path. This completes the induction, and the proof of the lemma.

4.9. Proposition. (Each connected component of) $\widetilde{T}$ is simply connected.

(That $\widetilde{T}$ is connected is asserted in Theorem 4.11.)

Proof. This is equivalent to the nonexistence of paths $V_{0}, \ldots, V_{r}$ with $r \geq 2$, no backtracks, and $V_{0}=V_{r}$. Suppose such a path exists. By possibly extending the path on both ends, one can easily arrange that $V_{0}, \ldots, V_{r}$ satisfies the conditions of Lemma 4.8. Therefore $V_{0}$ is an $F_{1}$ and the local ring $O$ of its special section dominates a closed point of $V_{r}$. But this is absurd, since $V_{r}=V_{0}$.

4.10. Action of $\mathrm{GA}_{2}$ on $\tilde{T}$. Let $S$ be a $W$-admissible model (hence a vertex in $\widetilde{T}$ ), and $\varphi$ an element of $\mathrm{GA}_{2}$. Extending $\varphi$ to an automorphism of $k(X, Y)$, we note that $\varphi$ carries the set of local rings of $W$ onto itself, and hence it carries the local rings of $S$ onto those of another $W$-admissible model $S^{\prime}$, with $S^{\prime}$ being the same type as $S$ (in the sense of 4.6). Thus $\mathrm{GA}_{2}$ acts on the set of $W$-admissible models. Clearly this action preserves adjacency in $\widetilde{T}$, so we have an action of $\mathrm{GA}_{2}$ on $\widetilde{T}$, since edges in $\widetilde{T}$ are determined by their end vertices

\subsection{Theorem.}

(1) $\widetilde{T}$ is a tree, and $\mathrm{GA}_{2}$ acts on $\widetilde{T}$ without version.

(2) A fundamental domain for the action is any geodesic

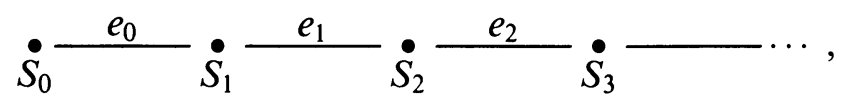

where $S_{0}$ is a $\mathbb{P}^{2}$ and $S_{i}$ is an $F_{i}$ for $i \geq 1$.

(3) The fundamental domain of (2) can be chosen so that $S_{0}$ is the standard $\mathbb{P}^{2}$ (see 3.3), and, $S_{i}$ is the standard $F_{i}$ (see 3.11). Therefore the stabilizer of $S_{0}$ is $A f$, and the stabilizer of $F_{j}$ is the subgroup

$$
\begin{aligned}
B A^{(i)}=\{(a X+b, c Y+g(X))\} \mid & a, c \in k^{*} ; b \in k ; \\
& g(X) \in K[X] \text { and } \operatorname{deg} g(X) \leq i)\} .
\end{aligned}
$$


Proof. It is clear that any $\mathbb{P}^{2}$ or $F_{i}$ is a translate, via some Cremona transformation, of the standard $\mathbb{P}^{2}$ or $F_{i}$. Since $A f=\mathrm{PGL}_{3} \cap \mathrm{GA}_{2}$ and $B A^{(i)}=$ $G^{(i)} \cap G A_{2}$ (see 3.9, 3.11), assertions (2) and (3) hold. As for (1), the simple connectivity of $\widetilde{T}$ was established in Proposition 4.9. It remains to show that $\widetilde{T}$ is connected.

The proof of the connectivity is relegated to the appendix, since it uses essentially the same techniques as several of the published proofs that $\mathrm{GA}_{2}$ is generated by linear and elementary automorphisms. We note, however, the elegance with which this framework leads to the proof of the complete structure theorem for $\mathrm{GA}_{2}$ (not just the fact that $\mathrm{GA}_{2}$ is generated by $A f$ and $B A$ ) as explained below in 4.12 .

4.12. Consequence. It follows from Theorem 4.11, and from the theory of groups acting on trees [15] that $\mathrm{GA}_{2}$ is the free product of the stabilizers of $S_{i}, i \geq 0$, amalgamated in pairwise fashion along the stablilizer of $e_{i}, i \geq 0$. The stabilizer of $e_{i}$ is the intersection of the stabilizers of $S_{i}$ and $S_{i+1}$, so one sees easily that the stabilizer of $e_{0}$ is $B$ (as defined in 2.3), and for $i \geq 1$ the stabilizer of $e_{i}$ is $B A^{(i)}$, since $B A^{(i)} \subset B A^{(i+1)}$. We thus have $\mathrm{GA}_{2}$ presented as the amalgamated free product

$$
A f *_{B} B A^{(1)} *_{B A^{(1)}} B A^{(2)} *_{B A^{(2)}} * B A^{(3)} * \cdots .
$$

Noting that $\bigcup_{i \geq 1} B A^{(i)}=B A$ (defined in 2.3), we readily obtain Theorem 2.4:

$$
\mathrm{GA}_{2}=A f *_{B} B A \text {, }
$$

as a consequence of the $\mathrm{GA}_{2}$-action on $\widetilde{T}$.

4.13. The simplified tree $T$. Our next step is to obtain from $\widetilde{T}$ a tree on which $\mathrm{GA}_{2}$ acts with a fundamental domain consisting of only one edge, yielding the decomposition (2). Of course, we know such a tree can be constructed abstractly, using Serre's theory [15]. Moreover, the tree Alperin produces in [1] has this property. But since $\widetilde{T}$ contains the apparatus which so naturally shows $\mathrm{GA}_{2}$ to be generated by $A f$ and $B A$, our task seems a worthy goal.

We proceed as follows: Consider the graph $Z$ obtained by removing from $\widetilde{T}$ all vertices of type zero (see 4.6) and all edges which have a type zero vertex as an end point. Form a new graph $T$ whose vertices consist of the type zero vertices of $T$ (i.e. the $\mathbb{P}^{2}$ 's) together with the connected components of $Z$. Connect a $\mathbb{P}^{2}$ with a component of $Z$ by an edge if they are connected by an edge in $\widetilde{T} . T$ is clearly a tree, since $\widetilde{T}$ is. This construction just amounts to shrinking the connected components of $Z$, which are subtrees of $\widetilde{T}$, down to points. Since the $\mathrm{GA}_{2}$ action on $\widetilde{T}$ preserves the types of the vertices, it is clear that elements of $\mathrm{GA}_{2}$ carry components of $Z$ to components of $Z$, and therefore $\mathrm{GA}_{2}$ acts on $T$. Moreover, it follows from (2) of Theorem 4.11 that a fundamental domain for the action of $\mathrm{GA}_{2}$ on $T$ consists of an edge

$$
\dot{S}_{0} \stackrel{e_{0}}{Z_{Z_{0}}}
$$

where $S_{0}$ is the $\mathbb{P}^{2}$ of (2) and $Z_{0}$ is the connected component of $Z$ containing the geodesic

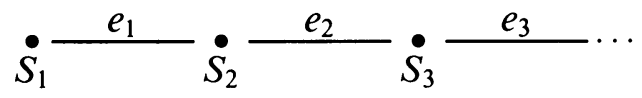


of (2). Taking $S_{0}$ to be the standard of $\mathbb{P}^{2}$ and $S_{i}, i \geq 1$, to be the standard $F_{i}$, the stabilizer of $S_{0}$ is $A f$ and the stabilizer of of $S_{i}, i \geq 1$, is $B A^{(i)}$. We claim that the stabilizer of $Z_{0}$ is $B A$. It clearly contains the stabilizers of $S_{1}, S_{2}, S_{3}, \ldots$, hence contains their union, which is $B A$. Conversely, suppose $\phi \in \mathrm{GA}_{2}$ is in the stabilizer of $Z_{0}$. Consider the geodesic in $\widetilde{T}$ from $S_{1}$ to $\phi \cdot S_{1}$, which necessarily lies within $Z_{0}$. Since a vertex of type $i \geq 1$ in $\widetilde{T}$ is adjacent to precisely one vertex of type $i+1$ (see 4.6), this geodesic is of the form

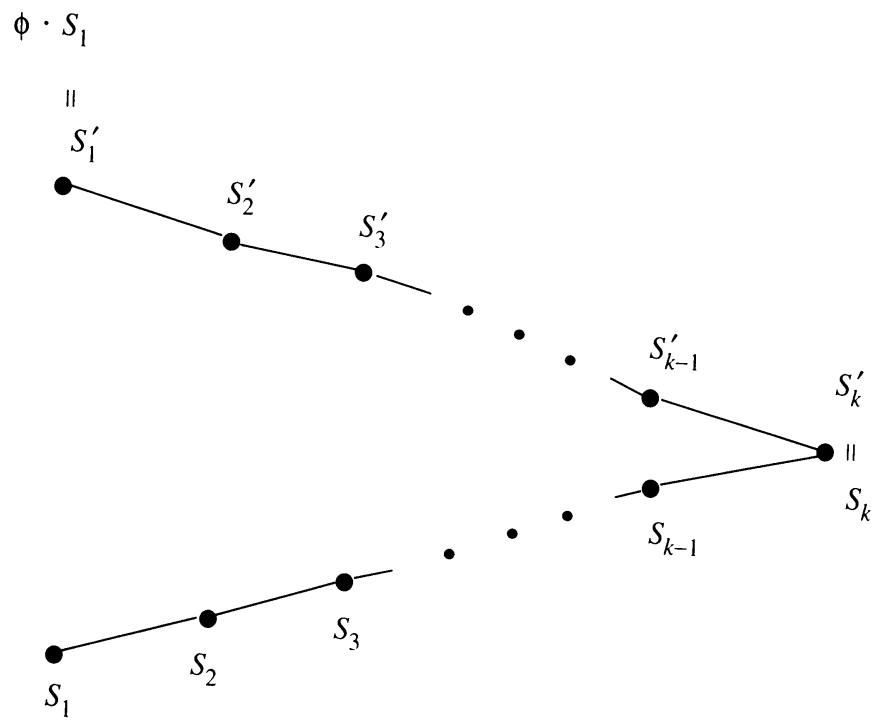

Moreover, it follows inductively that for $i=2, \ldots, k$ we must have $\phi \cdot S_{i}=S_{i}^{\prime}$, since $S_{i}^{\prime}$ and $\phi \cdot S_{i}$ are both the unique type $i$ vertex adjacent to $S_{i-1}^{\prime}$. Hence $\phi$ fixes $S_{k}$, so $\phi \in B A^{(k)} \subset B A$, and the claim is proved. Thus the tree $T$ has the desired properties.

4.14. $T$ as a tree of models. In order for us to establish the connection between the structure of $\mathrm{GA}_{2}$ and that of $\mathrm{Cr}_{2}$, it is useful to realize the tree $T$ as a tree whose vertices, like those of $\widetilde{T}$, are certain models in $k(X, Y)$. To do so, it will be necessary to allow as "models" certain $k$-schemes which are not varieties over $k$.

4.15. $\mathbb{P}^{1}$ 's in $k(X, Y)$. We consider $k$-schemes $R$ such that (1) $R \cong \mathbb{P}_{K}^{1}$ where $K$ is a field containing $k$ and (2) the function field of $R$ is $k(X, Y)$. Thus $k \subset K \subset k(X, Y)$ and $K$ is of transcendence degree 1 over $k$. It follows from Luroth's Theorem that $K=k(t)$, with $t$ transcendental over $k$. We can view such an $R$ as a certain collection of local rings in $k(X, Y)$, and it becomes clear that $\mathrm{Cr}_{2}$ acts transitively on the set of such $k$-schemes. We will call such a scheme a $\mathbb{P}^{1}$ in $k(X, Y)$. The standard $\mathbb{P}^{1}$, introduced in 3.8 , is the one for which $K=k(X)$.

4.16. The generic $\mathbb{P}^{1}$ associated to an $F_{n}(n \geq 1)$. If $S$ is an $F_{n}$ in $k(X, Y)$, the generic fiber $R$ of the map (unique for $n \geq 1$ ) $\pi: S \rightarrow \mathbb{P}_{k}^{1}$ is isomorphic to $\mathbb{P}_{K}^{1}$ where $K$ is the function field of $\mathbb{P}_{k}^{1}$. Thus we have subscheme $R$ (not open or closed) of $S$, canonical if $n \neq 0$, which is a $\mathbb{P}^{1}$ in 
$k(X, Y)$ (as defined in 4.15). For $n \geq 1$, we call $R$ the generic $\mathbb{P}^{1}$ associated to $S$.

4.17. The $\mathbb{P}^{1}$ associated to certain vertices of $T$. Let $S$ be an $F_{n}$, with $n \geq 1$, and let $p$ be any point on the section $\Delta_{n}$ of $S$. The elementary transformation $\operatorname{elm}_{p}$, which blows up $p$, then blows down the proper transform of its fiber, does not disturb the generic $\mathbb{P}^{1}$, call it $R$, of $S$. Therefore $R$ is also the generic $\mathbb{P}^{1}$ of the resulting $F_{n+1}$. It follows that if $S$ is an $F_{n}$ and $S^{\prime}$ is an $F_{m}$, both corresponding to vertices of $T$ and lying in the same connected component of $Z$, then $S$ and $S^{\prime}$ have the same generic $\mathbb{P}^{1}$, which we can therefore associate to this component. Since the components of $Z$ are vertices of $T$, all such vertices (i.e. those in the $\mathrm{GA}_{2}$ orbit of $Z_{0}$ ) have an associated $\mathbb{P}^{1}$ in $k(X, Y)$.

\subsection{Lemma. Distinct vertices in $T$ yield distinct $\mathbb{P}^{1}$ 's.}

Proof. Let $L$ and $L^{\prime}$ be distinct vertices in the $\mathrm{GA}_{2}$-orbit of $Z_{0}$. There is a unique geodesic in $T$ connecting $L$ and $L^{\prime}$, which is of the form
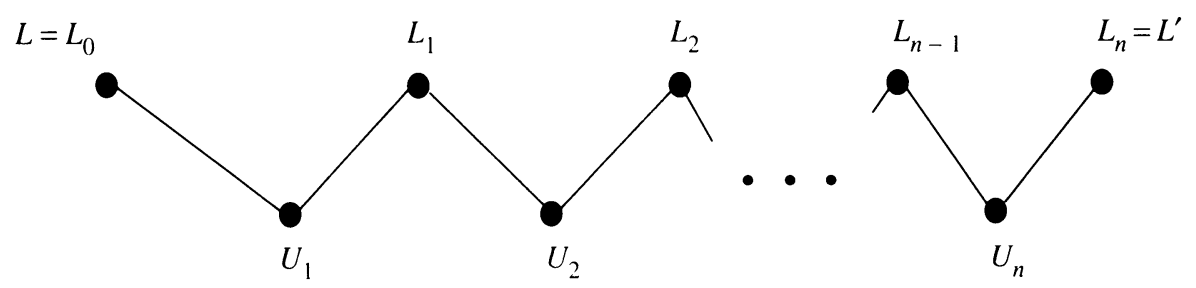

with $L_{0}, \ldots, L_{n}$ in the $\mathrm{GA}_{2}$-orbit of $Z_{0}$ and $U_{1}, \ldots, U_{n}$ in the $\mathrm{GA}_{2}$-orbit of $S_{0}$. This means, of course, that $U_{1}, \ldots, U_{n}$ are $\mathbb{P}^{2}$ 's. There exist unique representative models $V$ of $L=L_{0}$ and $V^{\prime}$ of $L^{\prime}=L_{n}$ which are $F_{1}$ 's such that $V$ is adjacent to $U_{1}\left(\cong \mathbb{P}^{2}\right)$ in $\widetilde{T}$ and $V^{\prime}$ is adjacent to $U_{n}$ in $\widetilde{T}$. The geodesic in $\widetilde{T}$ from $V$ to $V^{\prime}$ contains the vertices $U_{1}, \ldots, U_{n}$ of $\widetilde{T}$, and goes through the components $L_{1}, \ldots, L_{n-1}$ of $Z$. For $i=1, \ldots, n-1$, there exists a unique path with no backtracks through $L_{i}$ in $\widetilde{T}$ from $U_{i}$ to $U_{i+1}$. By juxtaposing these we obtain a path in $\widetilde{T}$ from $V$ to $V^{\prime}$ satisfying the conditions of Lemma 4.8. The lemma tells us that the local ring $O$ of the special section $\Delta$ on $V$ dominates a closed point on $V^{\prime}$. It follows that $O$ is the local ring of a point on $R$, the generic $\mathbb{P}^{1}$ of $V$ (and of $L$ ), but is not the local ring of a point on $R^{\prime}$, the generic $\mathbb{P}^{1}$ of $V^{\prime}$ (and of $L^{\prime}$ ). Therefore $R \neq R^{\prime}$.

4.19. $W$-admissible $\mathbb{P}^{1}$ 's. It is clear that a $\mathbb{P}^{1}$, call it $R$, in $k(X, Y)$ corresponds to a vertex of $T$ if and only if there exist, $F, G \in k[X, Y]$ such that $k[F, G]=k[X, Y]$ and such that $R=\operatorname{Spec} k(F)[G] \cup \operatorname{Spec} k(F)\left[G^{-1}\right] \cong$ $\mathbb{P}_{k(F)}^{1}$. If this is the case we say $R$ is a $W$-admissible $\mathbb{P}^{1}$.

4.20. $T$ as a tree of admissible models. We conclude from the above discussion that $T$ is a tree whose vertices consist of all $W$-admissible $\mathbb{P}^{2}$ 's and $\mathbb{P}^{1}$ 's in $k(X, Y)$. Given $S$ an admissible $\mathbb{P}^{2}$ and $R$ an admissible $\mathbb{P}^{1}, S$ and $R$ are adjacent vertices in $T$ if and only if there is a point $p$ at infinity in $S$ (with respect to $W$ ) such that $R$ is the generic $\mathbb{P}^{1}$ of the $F_{1}$ obtained by blowing 
up $S$ at $p$. The fundamental domain for the $\mathrm{GA}_{2}$-action on $T$ becomes

$$
E=\stackrel{\bullet}{S} \stackrel{R}{R}
$$

where $S$ and $R$ may be chosen to be $S=\operatorname{Proj} k[x, y, z], R=\operatorname{Spec} k(X)[Y] \cup$ $\operatorname{Spec} k(X)\left[Y^{-1}\right]=\mathbb{P}_{k(X)}^{1}$, where $X=x / z, Y=y / z$. One can reaffirm that the stabilizer in $\mathrm{GA}_{2}$ of $S$ is $A f$, and the stabilizer of $R$ consists of those $k$-automorphisms of $k[X, Y]$ which preserve the containment $k[X] \subset$ $k[X, Y]$-precisely $B A$.

We can summarize $4.14-4.20$ as follows:

4.21. Theorem. Let $T$ be the graph whose vertices consist of all $W$-admissible $\mathbb{P}^{2}$ 's and $\mathbb{P}^{1}$ 's, where $S, a \mathbb{P}^{2}$, is connected by an edge to $R, a \mathbb{P}^{1}$, precisely when $S$ has a point $p$ at infinity (with respect to $W$ ) such that $R$ is the generic $\mathbb{P}^{1}$ of the $F_{1}$ obtained by blowing up $S$ at $p$. Then

(1) $T$ is a tree, on which $\mathrm{GA}_{2}$ acts without inversion.

(2) A fundamental domain for the action is

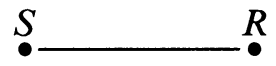

where $S$ is the standard $\mathbb{P}^{2}, R$ is the standard $\mathbb{P}^{1}$ (see $3.3,3.8$ ).

(3) The stabilizer of $S$ in $\mathrm{GA}_{2}$ is Af; the stabilizer of $R$ is $B A$.

From this theorem, the decomposition $\mathrm{GA}_{2}=A f *_{B} B A$ is immediate.

\section{THE SIMPLICIAL COMPLEX WHICH YIELDS THE STRUCTURE THEOREM FOR $\mathrm{Cr}_{2}$}

5.1. General theory. The amalgamated product group structure of $\mathrm{Cr}_{2}$ laid out in $\S 3$ reflects the fact that it acts on a simply connected 2-dimensional simplicial complex. This follows from a higher dimensional analogue of Serre's tree theory. (This is folklore amongst topologists, but see [18] or [17].) We wish to realize this space in such a way that its vertices again correspond to models in $k(X, Y)$, and such that it contains the tree $T$ of Theorem 4.21 as a subcomplex, compatibly with the containment $\mathrm{GA}_{2} \subset \mathrm{Cr}_{2}$.

5.2. Admissible models. Consider the set of models $S$ (model now means reduced, irreducible, separated $k$-scheme having function field $k(X, Y))$ satisfying one of these three properties:

(1) $S \cong \mathbb{P}_{k}^{2}$,

(2) $S \cong \mathbb{P}_{k}^{1} \times \mathbb{P}_{k}^{1}$, or

(3) $S \cong \mathbb{P}_{K}^{1}$ for some subfield $K$ of $k(X, Y)$ (necessarily of pure transcendence degree 1 over $k$ ).

Such a $k$-scheme $S$ will be called an admissible model. We say $S$ is a $\mathbb{P}^{2}, S$ is a $\mathbb{P}^{1} \times \mathbb{P}^{1}$, or $S$ is a $\mathbb{P}^{1}$ according to whether (1), (2), or (3), respectively, is satisfied.

5.3. The complex $C$. We construct a two-dimensional simplicial complex $C$ using as vertices the set of admissible models. We declare that three models $S$, a $\mathbb{P}^{2}, V$ a $\mathbb{P}^{1} \times \mathbb{P}^{1}$, and $R$ a $\mathbb{P}^{1}$, determine a face when there exist two distinct points $p$ and $q$ on $S$ such that (a) $V$ is the $\mathbb{P}^{1} \times \mathbb{P}^{1}\left(\cong F_{0}\right)$ obtained 
by blowing up $S$ at $p$ and $q$, then blowing down the proper transform of the line in $S$ containing $p$ and $q$, and (b) $R$ is the generic $\mathbb{P}^{1}$ of the $F_{i}$ obtained by blowing up $S$ at $p$.

Taking $S$ to be the standard $\mathbb{P}^{2}$ (see 3.3), $p=(0: 1: 0)$, and $q=(1: 0: 0)$, the resulting $V$ and $R$ are the standard $\mathbb{P}^{1} \times \mathbb{P}^{1}$ and $\mathbb{P}^{1}$, respectively (see 3.7, 3.8). Therefore these standard models form a face, which we will call the standard face in $C$.

5.4. Fundamental domain. It is clear from the construction of $C$ that $\mathrm{Cr}_{2}$ acts on $C$ without inverting any edge or rotating any face. Moreover, a fundamental domain for the action is given by any one face

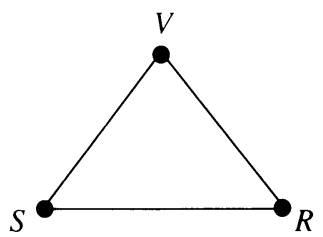

We may choose (1) to be the standard face (see 5.3). For this choice, the stabilizers of $S, V$, and $R$ are, respectively, the groups

$$
\begin{aligned}
& A_{1} \cong \mathrm{PGL}_{3}(k), \\
& A_{2} \cong\left(\mathrm{PGL}_{2}(k) \times \mathrm{PGL}_{2}(k)\right) \rtimes\langle\tau\rangle, \quad \text { and } \\
& A_{3} \cong \mathrm{PGL}_{2}(k) \ltimes \mathrm{PGL}_{2}(k(X)),
\end{aligned}
$$

identified as subgroups of $\mathrm{Cr}_{2}$ as in 3.3, 3.8, and 3.9.

\subsection{Theorem. The simplicial complex $C$ is 1-connected.}

Proof. This follows, using standard arguments, from the fact that (1) is a fundamental domain for the action of $\mathrm{Cr}_{2}$ on $C$, and the fact that $\mathrm{Cr}_{2}$ is the amalgamated free product of the stabilizers $A_{1}, A_{2}$, and $A_{3}$ along their pairwise intersections (Theorem 3.13). We sketch the proof.

To see that $C$ is connected, consider a face $F^{\prime}$, and let $F$ denote the standard face. We will show that there a path from $F$ to $F^{\prime}$. If $E$ is a face in $C$, then $E$ shares a vertex of $F$ if and only if $E=h F$ for some $h \in A_{1} \cup A_{2} \cup A_{3}$. Since $F$ is a fundamental domain, there exists $g \in \mathrm{Cr}_{2}$ such that $g F=F^{\prime}$. Let $g=g_{1} \cdots g_{r}$ be a factorization of $g$ such that $g_{1}, \ldots, g_{r} \in$ $A_{1} \cup A_{2} \cup A_{3}$. Consider the sequence of faces $F=F_{0}, F_{1}, \ldots, F_{r}=F^{\prime}$, where $F_{i}=g_{1} \cdots g_{i} F, i=0, \ldots, r$. For $i=0, \ldots, r-1, g_{i+1} F$ touches $F$, as previously observed, hence $F_{i+1}$ touches $F_{i}$ (translating by $g_{1} \cdots g_{i}$ ). Therefore the union of these faces contains a path from $F$ to $F^{\prime}$. This shows $C$ is connected.

To show simple connectivity, we associate a loop to a sequence of faces $F_{0}, \ldots, F_{r}$ such that $F_{i}$ and $F_{i+1}$ have a common vertex, for $i=0, \ldots, r-1$, and such that $F_{0}=F_{r}$. We may assume that $F_{0}$ and $F_{r}$ are the standard face. Thus we have a sequence $g_{1}, \ldots, g_{r} \in A_{1} \cup A_{2} \cup A_{3}$ such that $F_{i}=g_{1} \cdots g_{i} F_{0}$, for $i=1, \ldots, r$. Since $F_{r}=F_{0}$, we see that $g_{1} \cdots g_{r} F_{0}$ is in the stabilizer of $F_{0}$, which is $A_{1} \cap A_{2} \cap A_{3}$. The fact that $\mathrm{Cr}_{2}$ is the pairwise amalgamated product of $A_{1}, A_{2}$, and $A_{3}$ (see Theorem 3.13, and $\S 5.4$ ) implies that the sequence $g_{1}, \ldots, g_{r}$ can be transformed into the sequence consisting only of 
the element $g=g_{1} \cdots g_{r}$ by a series of the following types of changes:

(a) replace a sequence entry $h$ by $h^{\prime} h^{\prime \prime}$, where $h, h^{\prime}, h^{\prime \prime} \in A_{i}$ and $h=$ $h^{\prime} h^{\prime \prime}$.

(b) replace two successive entries $h^{\prime}, h^{\prime \prime}$ by $h$, where $h^{\prime}, h^{\prime \prime} \in A_{i}$ and $h=h^{\prime} h^{\prime \prime}$.

An alteration of either of these types replaces the path $F_{0}, \ldots, F_{r}$ by a homotopic path. Thus the path contracts into the face $F_{0}$. This concludes the proof.

5.6. The $\mathrm{Cr}_{2}$ complex $C$ contains the $\mathrm{GA}_{2}$ tree $T$. If we choose for the fundamental domain $F$ of $C$ the standard face, (1) and let $W$ be the standard $\mathbb{A}^{2}$, which is the complement in $S$ of the line containing $p=(0: 1: 0)$ and $q=(1: 0: 0)$, then the tree $T$ of $\S 4$ is the union of the $\mathrm{GA}_{2}$ translates of the edge

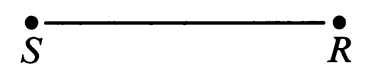

of $F$. Thus the complex $C$ contains the tree $T$ as a subcomplex, and the face $F$ contains the fundamental domain $E$ of $T$, as follows:

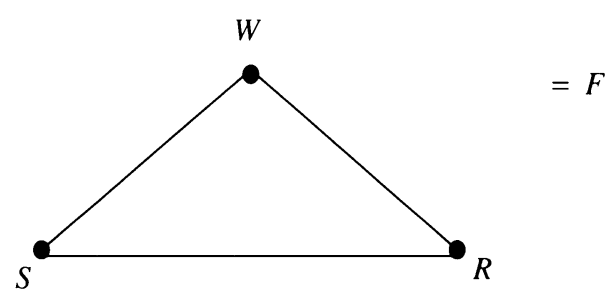

UI

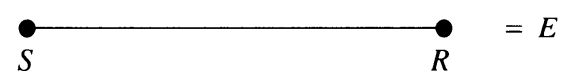

\section{APPENDIX}

6.1. Here we sketch a proof of the connectivity of the graph $\tilde{T}$, which completes the proof of Theorem 4.11. The reader is assumed to be familiar with the basic facts about birational maps between smooth surfaces, and with intersection numbers and their behavior under the blowing up of points.

As before, all varieties discussed are assumed to be $k$-varieties, where $k$ is a fixed algebraically closed field.

6.2. Lemma. Let $V$ be a nonsingular complete surface and $\omega: V \rightarrow \mathbb{P}_{k}^{1} a$ morphism making $V$ a ruled surface (i.e., for some open set $U \subseteq \mathbb{P}_{k}^{1}, \omega^{-1}(U) \cong$ $\left.\mathbb{P}_{k}^{1} \times U\right)$. Then each fiber of $\omega$ contains a component having self-intersection $\geq-1$.

Proof. Let $W=\mathbb{P}_{k}^{1} \times \mathbb{P}_{k}^{1}$. Taking $\pi: W \rightarrow \mathbb{P}_{k}^{1}$ to be one of the projections, 
there exists a birational map $\gamma: V \rightarrow W$ such that

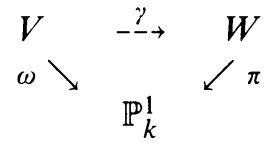

commutes.

Suppose $D$ is a fiber of $\omega$ all of whose components have self-intersection $\leq-2$. We will arrive at a contradiction by resolving $\gamma$ to a morphism. Note that the commutativity of (1) implies that any curve of $V$ which maps to a point in $W$ must be contained in a fiber of $\omega$, and the same must hold if we blow up a point of $V$ and replace $V$ by the surface thus obtained. By successively blowing up points of indeterminacy for $\gamma$ which do not lie on $D$, we may assume $\gamma$ is defined at all points not lying on $D$. (These blow-ups do not alter the self-intersection of the components of $D$.)

First we argue that $\gamma$ is not everywhere defined. Since $\left(D^{2}\right)=0, D$ has more than one component, and the image of $D$ under $\gamma$ lies within a fiber $C$ of $\pi$. If $\gamma$ were a morphism it would factor into a sequence of blowing downs, and $D$ would contain a component $E$ with $\left(E^{2}\right)=-1$, in violation of our assumption.

So let $x$ be a point on $D$ at which $\gamma$ is not defined. We proceed to resolve $\gamma$ by blowing up $x$, creating a new surface $V_{1}$ with exceptional curve $E_{1}$, and induced rational map $\gamma_{1}: V_{1} \rightarrow W . V_{1}$ is a ruled surface and the proper transform $D_{1}$ of $D$ is a fiber whose components are the proper transforms of the components of $D$, together with $E_{1}$. Note that all the components of $D_{1}$, except $E_{1}$, have self-intersection $\leq-2$, and $\left(E_{1}^{2}\right)=-1$.

Case 1. $\gamma_{1}$ is defined at all points of $E_{1}$. In this case we must have $\gamma_{1}\left(E_{1}\right)=C$; for if $\gamma_{1}\left(E_{1}\right)$ is a point, then $\gamma_{1}$ factors through the blowing down of $E_{1}$, contradicting the fact that $x$ was a point of indeterminacy for $\gamma$. It easily follows that $\gamma_{1}$ is defined everywhere. For if not, we could resolve $\gamma_{1}$ to a morphism by blowing up more points, and the last exceptional curve must necessarily map into $C$ (otherwise the last blow-up would have been unnecessary). However a birational morphism of surfaces cannot carry two distinct curves onto the same curves. So $\gamma_{1}$ is a morphism, with $D_{1}$ mapping into $C$ and $E_{1}$ mapping onto $C$. Since $\gamma_{1}$ is the product of blowing downs, and all components of $D_{1}$ other than $E_{1}$ collapse to a point, one of them must have self-intersection -1 . This is a contradiction.

Case 2. There exists a point $x_{1} \in E_{1}$ which is a point of indeterminacy for $\gamma_{1}$. We blow up $x_{1}$ to obtain a surface $V_{2}$ with exceptional curve $E_{2}$ and the induced birational map $\gamma_{2}: V_{2} \rightarrow W . V_{2}$ has fiber $F_{2}$ over $\mathbb{P}_{k}^{1}$ which is the total transform of $D_{1}$; all of its components, other than $E_{2}$, have selfintersection $\leq-2$. If $\gamma_{2}$ is defined along $E_{2}$ we reach the same contradiction as in Case 1. Otherwise we again blow up a point of indeterminacy on $E_{2}$. This process must end (in a contradiction) when $\gamma$ is finally resolved to a morphism.

6.3. To show that $\widetilde{T}$ is connected, it suffices to show that, given any two $W$ admissible $\mathbb{P}^{2}$ 's, $S_{0}$ and $S$, there is a path in $\widetilde{T}$ from $S_{0}$ to $S$. (Recall that $W$ is the standard $\mathbb{A}^{2}$ (see 2.1).) We may assume that $S_{0}$ is the standard $\mathbb{P}^{2}$ (see 3.3). Let $P, Q \in k[X, Y]$ be a system of variables which define straight 
lines in $W$ relative to the embedding $W \subset S \cong \mathbb{P}^{2}$. If $P$ and $Q$ are both linear polynomials in $X$ and $Y$, then $S=S_{0}$ and there is nothing to prove. In the general case, let $d=\operatorname{deg} P$; we will reduce to the case $d=1$.

6.4. Let $L$ be the line at infinity (relative to $W$ ) in $S_{0}$, and let $C$ be the closure in $S_{0}$ of the curve in $W$ defined by $P$. Since $C \cap W \cong \mathbb{A}_{k}^{1}, C$ has one point $x$ on $L$, and it is a one place point, i.e., the closure of $C \cap W$ in any smooth complete surface containing $W$ will always have one point at infinity (relative to $W$ ), and that point will have only one tangential direction. Note that $(L \cdot C)=d$, by Bezout's Theorem. Let $d_{1}=$ mult $_{x} C$, the multiplicity of $x$ on $C$. Since $d>1, L$ must be tangent to $C$ at $x$; for otherwise $d=d_{1}$, and letting $H$ be the line on $S_{0}$ tangent to $C$ at $x$, we have $(H \cdot C)>d$, violating Bezout's Theorem. Let $m=d-d_{1}$ and write $d=n m+r$ with $0 \leq r<m$. We will eventually show that $r=0$, i.e., $m \mid d$. (This is the crux of the proof.)

6.5. We blow up $S_{0}$ at $x$ to obtain a $W$-admissible surface $S_{1} \cong F_{1}$. Note that $S_{1}$ is a vertex in $\widetilde{T}$ adjacent to $S_{0}$. Let $E_{1}$ be the resulting exceptional curve on $S_{1}$, and let $L_{1}$ and $C_{1}$ be, respectively, the proper transforms of $L$ and $C$. The complement of $W$ in $S_{1}$ is $E_{1} \cup L_{1}$, and $C_{1}$ intersects this complement at the point where $E_{1}$ and $L_{1}$ intersect; call this point $x_{1}$. We have $\left(E_{1} \cdot C_{1}\right)=d_{1}=d-m$, and an easy argument shows $\left(L_{1} \cdot C_{1}\right)=m$.

6.6. If $d_{1}>m$, then $E_{1}$ is tangent to $C_{1}$ at $x_{1}$,

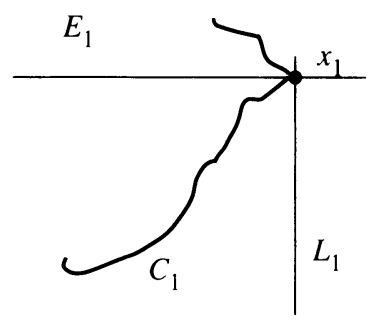

and $m=$ mult $_{x_{1}} C_{1}$. In this case, we blow up $x_{1}$, separating $E_{1}$ and $L_{1}$, and contract the proper transform of $L_{1}$ to obtain a $W$-admissible surface $S_{2} \cong F_{2}$, adjacent to $S_{1}$ in $\widetilde{T}$. Let $E_{2}$ and $C_{2}$ be the proper transform of $E_{1}$ and $C_{1}$ in $S_{2}$, and let $L_{2}$ be the proper transforms of the exceptional curve obtained from blowing up $x_{1}$. The complement of $W$ in $S_{2}$ is $E_{2} \cup L_{2}$, and $C_{2}$ intersects this complement at the point $x_{2}$ where $E_{2}$ and $L_{2}$ intersect. We have $\left(E_{2} \cdot C_{2}\right)=d_{1}-m=d-2 m$ and $\left(L_{2} \cdot C_{2}\right)=m$.

6.7. If $d-2 m>m$, then $E_{2}$ is tangent to $C_{2}$ at $x_{2}$, and $m=$ mult $_{x_{2}} C_{2}$. Repeating this process $n$ times, where $d=n m+r$ (possibly proceeding through the situation $d-(n-1) m=m$, in case $m \mid d)$, we obtain a $W$-admissible surface $S_{n} \cong F_{n}$, connected to $S_{0}$ in $\widetilde{T}$ by the path $S_{0}, S_{1}, \ldots, S_{n}$. The complement of $W$ in $S_{n}$ is $E_{n} \cup L_{n}$, and $C_{n}$ is the proper transform of $C$. Letting $x_{n}$ be the point where $E_{n}$ and $L_{n}$ intersect, we have $\left(E_{n} \cdot C_{n}\right)=d-m n=r$ and $\left(L_{n} \cdot C_{n}\right)=m$. We have $0 \leq r<m$ and $x_{n}$ lies on $C_{n}$ if and only if $r \neq 0$. In either case, the point at infinity (with respect to $W$ ) of $C_{n}$ lies on $L_{n}$. Call this point $q$. Then $q=x_{n}$ if and only if $r \neq 0$. Note that these conditions must hold if $n=1$ (a situation we later deem impossible). 
6.8. We claim that $L_{n}$ is not tangent to $C_{n}$ at $q$. To prove this, suppose $L_{n}$ and $C_{n}$ do meet tangentially, and consider the rational map $\omega: S_{n} \rightarrow \mathbb{P}_{k}^{1}$ induced by the containment $k(F) \subset k(X, Y)$. The divisor of $F$ on $S_{n}$ is easily seen to be $(F)=C_{n}-d L_{n}-m E_{n}$; it follows that $q$ is the only point of indeterminacy for $\omega$, as it is the only point where neither $F$ nor $F^{-1}$ is defined. We proceed to resolve $\omega$ to a morphism $\tilde{\omega}: V \rightarrow \mathbb{P}_{k}^{1}$ by blowing up points of indeterminacy, beginning with $q$. In this process, each time we blow-up we get an exceptional curve $E$ such that either (1) $E$ contains at least one point of indeterminacy, or (2) $\tilde{\omega}$ is defined at all points of $E$. In case (1), the proper transform of $E$ on $V$ has self-intersection $\leq-2$ ( since $\left(E^{2}\right)=-1$ and blowing up the point(s) of indeterminacy on $E$ causes its self-intersection to drop). In case (2) the image of $E$ under $\omega$ is all of $\mathbb{P}_{k}^{1}$ (for if its image were a point, the last blow-up would have been unnecessary). It follows that all components of the complement of $W$ in $V$, excepting certain curves which map onto $\mathbb{P}^{1}$, have self-intersection $\leq-2$. This is due to the fact that $C_{n}$ is tangent to $L_{n}$ at $q$, and $\left(L_{n}^{2}\right)=0$. Thus at least two blow-ups occur on $L_{n}$ or its proper transforms, so that its proper transform on $V$ also has selfintersection $\leq-2$. As for $E_{n}$, we have $\left(E_{n}^{2}\right)=-n$, and the only problem could arise when $n=1$. But in this case we have noted that $q \in E_{n}$, so that the proper transform of $E_{n}$ has self-intersection $\leq 2$ as well. Let $p \in \mathbb{P}_{k}^{1}$ be the point at which $F$ has its pole, and note that $\omega^{-1}(p)$ lies in the compliment of $W$ in $V$, but obviously does not contain the curve(s) which map onto $\mathbb{P}_{k}^{1}$. Hence this fiber consists of components having self-intersection $\leq-2$, in violation of Lemma 6.2. The claim is proved.

6.9. We conclude that $r=0$, i.e. $m \mid d$. For if not then $q=x_{n}$, as was seen in 6.7, and the fact that $\left(L_{n} \cdot C_{n}\right)=m>r=\left(E_{n} \cdot C_{n}\right)$ would say that $L_{n}$ is tangent to $C_{n}$ at $q$, which we know from 6.8 is not the case. Moreover, it follows from this and from 6.7 that $n>1$. The situation is as depicted below:

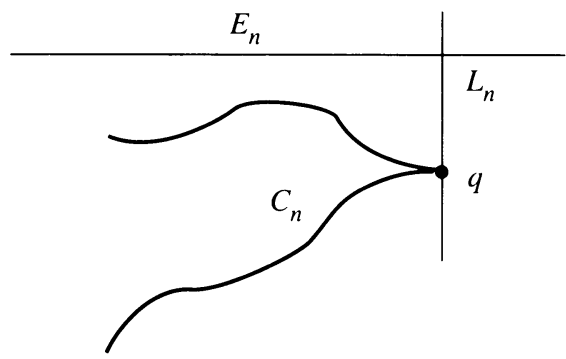

Note that $m=\operatorname{mult}_{q} C_{n}$. Now we blow up $q$, separating $C_{n}$ and $L_{n}$, and blow down the proper transform of $L_{n}$ to obtain a $W$-admissible surface $S_{n-1}^{\prime} \cong$ $F_{n-1}$, adjacent to $S_{n}$ in $\widetilde{T}$. Letting $E_{n-1}^{\prime}$ and $C_{n-1}^{\prime}$ be the proper transforms of $E_{n}$ and $C_{n}$, and letting $L_{n-1}^{\prime}$ be the new fiber at infinity, we have $\left(L_{n-1}^{\prime}\right.$. $\left.C_{n-1}^{\prime}\right)=m$, and $\left(E_{n-1}^{\prime} \cdot C_{n-1}^{\prime}\right)=0$. The argument of 6.8 shows that $L_{n-1}^{\prime}$ is not tangent to $C_{n-1}^{\prime}$ at their point of intersection, call it $q_{n-1}$, provided $n-1 \geq 2$. Thus mult ${ }_{q_{n-1}} C_{n-1}^{\prime}=m$. We repeat this process and thereby continue along a path in $\widetilde{T}$ to arrive at a surface $S_{1}^{\prime} \cong F_{1}$ and the situation depicted below: 


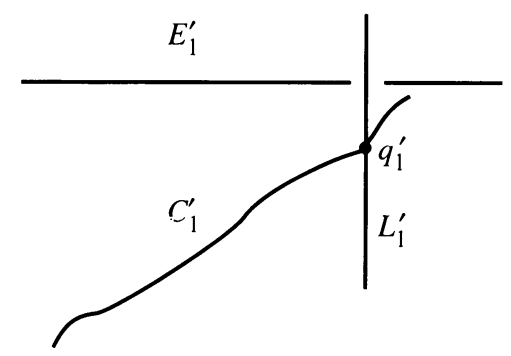

with $\left(C_{1}^{\prime} \cdot L_{1}^{\prime}\right)=m$ (but we no longer know, at this step, that $L_{1}^{\prime}$ is not tangent to $C_{1}^{\prime}$ at $\left.q_{1}^{\prime}\right)$. Now we blow down $E_{1}^{\prime}$ to obtain a $W$-admissible surface $S_{0}^{\prime} \cong \mathbb{P}_{k}^{2}$, with $C^{\prime}$ the proper transform of $C_{1}^{\prime}$ and $L^{\prime}$ the proper transform of $L_{1}^{\prime}$. $L^{\prime}$ is the line at infinity with respect to $W$, and $\left(L^{\prime} \cdot C^{\prime}\right)=m$. Therefore $C^{\prime}$ is a curve of degree $m$ on $S_{0}^{\prime}$, and if we write $F$ as a polynomial in variables $X^{\prime}$, $Y^{\prime}$ which define straight lines in $S_{0}^{\prime}$, its degree is $m$. Since $m<d$ the degree of $F$ has been lowered.

The path in $T$ which has been traversed is illustrated below:
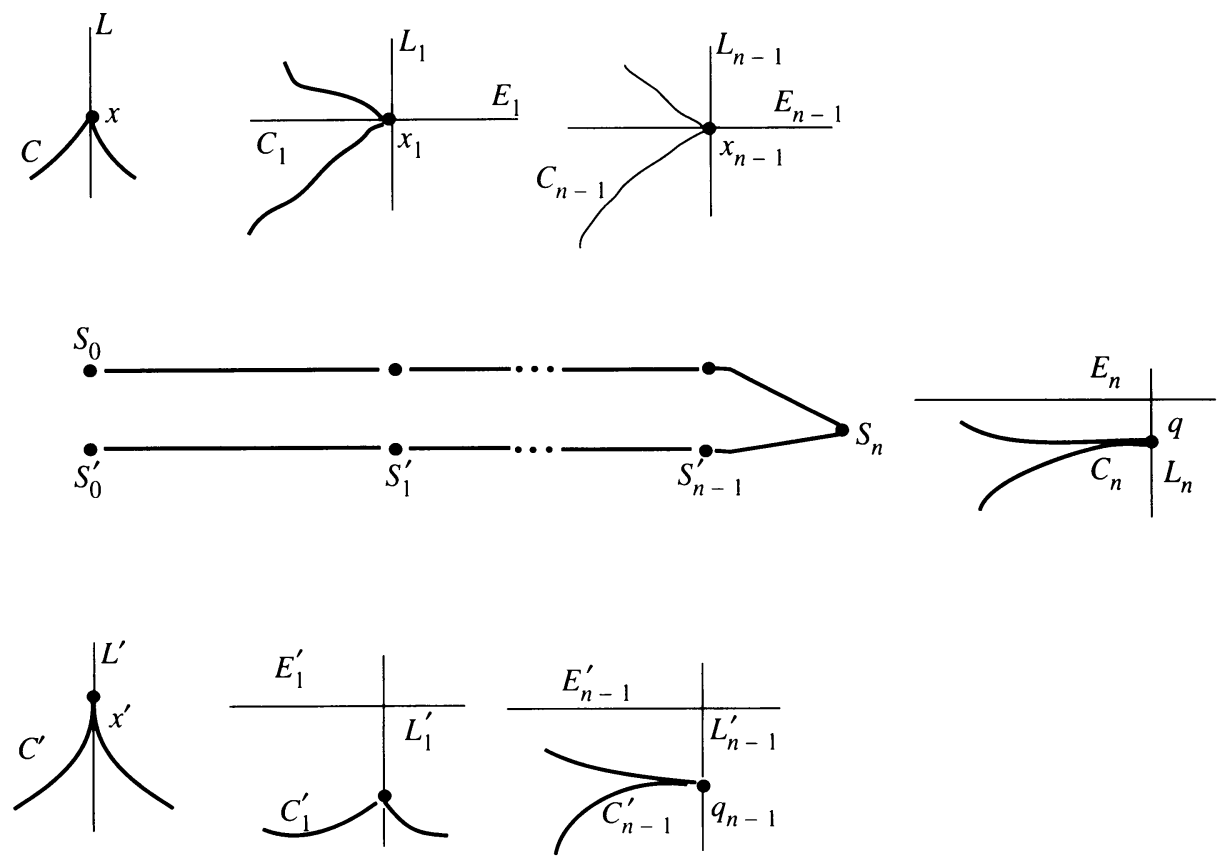

6.10. We can repeat this procedure until $d=1$. Now consider $e=\operatorname{deg} G$, and assume $e>1$. We will perform the same operation as above, with respect to $G$ instead of $F$. So now let $C$ be the curve defined by $G$, and let $D$ be the curve defined by $F$, which is a straight line in $S_{0}$, since $d=1$. We again let $L$ be the line at infinity. Since $C$ and $D$ meet once on $W$, they intersect $e-1$ times at their common point at infinity. We now trace the proper transform of $D$ through the path illustrated in 6.9. Note that the proper transform of $D$ on $S_{1}$ is a fiber of $F_{1}$, and this holds at each vertex in the path, through $S_{1}^{\prime}$. Thus the proper transform $D^{\prime}$ of $D$ in $S_{0}^{\prime}$ is again a straight line. Hence as a polynomial in $X^{\prime}$ and $Y^{\prime}, F$ is still linear, and the degree of $G$ has been 
lowered. When we finally achieve $d=e=1$, we have $S_{0}=S$, and the proof is complete.

\section{BIBLIOGRAPHY}

1. R. C. Alperin, Homology of the group of automorphisms of $k[x, y]$, J. Pure Appl. Algebra 15 (1979), 109-115.

2. G. Castelnuovo, La trasformazioni generatrici del gruppo Cremoniano nel piano, Atti Acad. Sci. Torino 36 (1901), 861-874.

3. W. Dicks, Automorphisms of the polynomial ring in two variables, Publ. Mat. 27 (1983).

4. M. H. Gizatullin and V. I. Danilov, Automorphisms of affine surfaces. I, Math. USSR-Izv. 9 (1975), no. 3, 493-534.

5. __, Automorphisms of affine surfaces. II, Math. USSR-Izv. 11 (1977), no. 1, 51-98.

6. H. P. Hudson, Cremona transformations, Cambridge University Press, Cambridge, 1927.

7. V. A. Iskovskikh, Birational automorphisms of three-dimensional algebraic varieties, J. Soviet Math. 13 (1980), 815-868.

8. __, Generators and relations in a two-dimensional Cremona group, Vestnik Moskov. Univ. Ser. I. Mat. Mekh. 38 (1983), no. 5, 43-48.

9. __ Proof of a theorem on relations in the two dimensional Cremona group, Uspekhi Mat. Nauk 40 (1985), no. 5 (245), 255-256.

10. H. W. E. Jung, Über ganze birationale Transformationen der Ebene, J. Reine Angew. Math. 184 (1942), 161-174.

11. J. H. McKay and S. Wang, An elementary proof of the automorphism theorem of a polynomial ring in two variables, J. Pure Appl. Algebra 52 (1988), 91-102.

12. M. Nagata, On automorphism groups of $k[X, Y]$, Kyoto Univ. Lectures in Math. No. 5, Kinokuniya-Tokyo, 1972.

13. __, Our rational surfaces. I, Mem. College Sci. Kyoto Univ. 32 (1960).

14. M. Noether, Zur Theorie der eindeutigen Ebenentransformationen, Math. Ann. 5 (1972), 635-639.

15. J.-P. Serre, Arbes, amalgames, et $\mathrm{SL}_{2}$, Astérisque 46 (1977).

16. I. R. Shafarevich, Algebraic surfaces, Proc. Steklov Inst. Math. 75 (1965).

17. C. Soulé, Groupes opérant sur un complexe simplicial avec domaine fondamental, C. R. Acad. Sci. Paris Sér. A 276 (1973), 607-609.

18. R. G. Swan, Generators and relations for certain special linear groups, Adv. in Math. 6 (1971), 1-77.

19. W. Van der Kulk, On polynomial rings in two variables, Nieuw Arch. Wisk. 1 (1953), 33-41.

20. D. Wright, Algebras which resemble symmetric algebras, Thesis, Columbia University, 1975.

Department of Mathematics, Washington University, St. Louis, Missouri 63130 